\title{
Quelques nOUVelles espèces de MYXOSPORIDIes, DU GeNRE CERATOMYXa THÉlOHAN, 1895 parasites des poIsSONS marins du SÉnÉGal, Afrique de l'OUest
}

\author{
KPATCHA T.K.*, DIEBAKATE C.*, FAYE N.* \& TOGUEBAYE B.S.*
}

Summary : NEW SPECIES OF MYXOSPORIDIA OF THE GENUS CERATOMYXA THÉLOHAN, 1895 PARASITES OF MARINE FISHES OF SEnegal, West AFrica

Seven species of Ceratomyxa were found in the gall-bladder of marine fishes collected from the coats of Senegal. These species were: C. acanthuri n. sp. from Acanthurus monroviae, C. fistulariae n. sp. from Fistularia petimba, C. lagocephali n. sp. from Lagocephalus laevigatus, C. syacii n. sp. from Syacium micrurum, C. trachinocephali n. sp. from Trachinocephalus myops., C. trichiuri n. sp. from Trichiurus lepturus and C. truncata Thélohan, 1895 from Sardinella maderensis and Sardinella aurita.

KEY WORDS : Myxosporidia, Ceratomyxa, parasites, marine fishes, Senegal West Africa.

\section{INTRODUCTION}

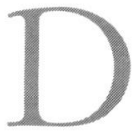

e nombreuses Myxosporidies appartenant au genre Ceratomyxa Thélohan, 1892 sont parasites des poissons marins (Kudo, 1919; Schulman, 1966; Lubat et al., 1989; Lom, 1990; Lom et Dykovà, 1992). La majorité de ces parasites vivent dans la vésicule biliaire de leurs hôtes et ne sont pas pathogènes. La plus importante espèce connue pour ses effets pathogènes est Ceratomyxa shasta. Elle parasite l'intestin de Salmonidae (Bartholomew et al., 1989; Lom et Dykovà, 1992).

En Afrique, quelques travaux ont été réalisés sur ces parasites. On peut citer par exemple ceux de Kudo (1919), Fantham (1930), Baker (1963), Peters (1971), Abolarin (1971, 1974), Siau (1971), Paperna (1973), Dubina \& Isakov (1976), Berrebi (1980), Fomena et al. (1985, 1993), Fomena \& Bouix (1986, 1987), Faisal \& Shalaby (1987), Obiekezie et Okaeme (1987, 1990), Obiekezie \& Enyenihi (1988), Sakiti et al. (1991), Obiekezie \& Schmahl (1993), Kostoïngué \& Toguebaye (1994), Kabré et al. (1995). Dans le présent travail, il est signalé pour la première fois la présence de Ceratomyxa chez des poissons marins des côtes sénégalaises

\footnotetext{
* Laboratoire de Parasitologie (J.E.R. 3002 associée à l'AUPELF/UREF), Département de Biologie Animale, Faculté des Sciences et Techniques, Université C. A. Diop de Dakar, Dakar, Sénégal.

Correspondance : Dr Bhen S. Toguebaye, Faculté des Sciences et Techniques, Dakar, Sénégal. Tél./Fax: (221) 252529.
}

\section{Résumé :}

L'étude de la faune myxosporidienne des poissons marins des côtes sénégalaises a permis de trouver sept espèces du genre Ceratomyxa parasitant la vésicule biliaire de ces poissons. Ce sont: C. acanthuri n. sp. chez Acanthurus monroviae, C. fistulariae n. sp. chez Fistularia petimba, C. lagocephali n. sp. chez Lagocephalus laevigatus, C. syacii n. sp. chez Syacium micrurum, C. trachinocephali n. sp. chez Trachinocephalus myops., C. trichiuri n. sp. chez Trichiurus lepturus et C. truncata Thélohan, $1895 \mathrm{chez}$ Sardinella maderensis et Sardinella aurita.

MOTS CLÉS : Myxosporidies, Ceratomyxa, parasites, poissons marins, Sénégal, Afrique de l'Ouest.

\section{MATÉRIEL ET MÉTHODES}

922 poissons marins du Sénégal appartenant à 37 familles, 51 genres et 63 espèces ont été examinés. Ces poissons proviennent de cinq localités : Kayar sur la grande côte, Mbour et Joal-Fadiouth sur la petite côte, Dakar (ports de pêche artisanale de Hann, Ouakam, et Soumbédioune) et Bargny. Des frottis frais de divers organes de chaque poisson ont été observés au microscope photonique pour rechercher les Myxosporidies. Les spores vivantes observées sont photographiées, mesurées à l'aide d'un micromètre oculaire et dessinées à l'aide d'un tube à dessin. Des frottis destinés à être conservés ont été réalisés. Ces frottis sont séchés, fixés au méthanol, colorés au Giemsa, déshydratés à l'acétone et montés au baume de Canada.

\section{RÉSULTATS}

\section{CERATOMYXA TRACHINOCEPHALI N. SP.}

Hôte: Trachinocephalus myops (Forster, 1801) (Synodontidae).

Site d'infestation : vésicule biliaire

Prévalence : $28,5 \%(12 / 42)$

\section{DESCRIPTION}

Les spores de cette espèce sont arquées (fig. 1 et 8 ). Elles ont une largeur de 49,66 $\pm 0,7$ (48-50) $\mu \mathrm{m}$ et un 
diamètre sutural de 11,5 \pm 0,8 (10-12) $\mu \mathrm{m}$. Les capsules polaires sont sphériques et ont un diamètre de 2,7 $\pm 0,4$ (2-3) $\mu \mathrm{m}$. La ligne de suture est nettement visible en microscopie photonique (fig. 1). Les extrémités des valves sont arrondies.

\section{Discussion}

Par la largeur de ses spores, cette espèce ressemble à Ceratomyxa acadiensis Mavor, 1915 parasite de la vésicule biliaire de Urophycis chuss, Zoarces angularis et Pseudopleuronectes americanus du Canada (Kudo, 1919). Mais elle en diffère par le diamètre sutural de ses spores et par le diamètre de ses capsules polaires. Par ailleurs, les extrémités des valves de $C$. acadiensis présentent chacune un long filament. Il faut aussi noter que c'est la première fois qu'une Myxosporidie du genre Ceratomyxa est trouvée chez les Synodontidae. Nous pensons que l'espèce étudiée ici est nouvelle et proposons de la nommer Ceratomyxa trachinocephali du nom de genre de son hôte.

\section{CERATOMYXA ACANTHURI N. SP.}

Hôte : Acanthurus monroviae Steindachner, 1876 (Acanthuridae)

Site d'infestation : vésicule biliaire

Prévalence : $36,3 \%(12 / 33)$

\section{DESCRIPTION}

Les pansporoblastes sont sphériques, mesurent 19 à $22 \mu \mathrm{m}$ de diamètre et contiennent deux spores (fig. 2). Les spores (fig. 2 et 9) ont des extrémités arrondies et mesurent 16,57 $\pm 0,9$ (16-18) $\mu \mathrm{m}$ de large; leur diamètre sutural est de $10,54 \pm 0,8(10-12) \mu \mathrm{m}$. Les capsules polaires sont sphériques et occupent la région antérieure de la spore; elles ont un diamètre de $2,75 \pm 0,6(2-3,20) \mu \mathrm{m}$. Très souvent une valve est plus courte et plus haute que l'autre (fig. 2).

\section{DISCUSSION}

Par la forme et la largeur de ses spores, cette espèce se rapproche de Ceratomyxa sp. de la vésicule biliaire de Gobius bucchichi des côtes du Monténegro (Adriatique Sud) décrit par Lubat et al., 1989 et de Ceratomyxa hungarica trouvé par Molnàr (1992) chez Proterorhinus marmoratus d'eau douce de Budapest en Hongrie.

Ceratomyxa sp. de G. bucchichi forme des spores mesurant 16,95 (16,5 à 17,25) $\mu \mathrm{m}$ de large, $7,5(2,25$ à 8) $\mu \mathrm{m}$ de diamètre sutural et possèdant des capsules polaires de $2,5 \mu \mathrm{m}$ de diamètre et des valves inégales à extrémités arrondies. Ces spores sont légèrement arquées (Lubat et al., 1989)

Ceratomyxa hungarica parasite les reins de son hôte. Ses spores mesurent 18,8 (18,5-19,5) $\mu \mathrm{m}$ de large avec un diamètre sutural de 10,1 (10,0-10,5) $\mu \mathrm{m}$. Les cap- sules polaires sont sphériques et ont un diamètre de $4,8(4,5-5,0) \mu \mathrm{m}$. Le filament polaire est visible chez les spores vivantes; il décrit six tours de spire. Les valves sont égales ( Molnàr, 1992).

L'espèce de $A$. monroviae se distingue néanmoins de ces deux espèces soit par le diamètre sutural de ses spores, soit par la forme de ses valves, soit par le diamètre de ses capsules polaires, soit encore par le biotope et la localisation géographique de son hôte. Nous pensons qu'elle est nouvelle et proposons de la nommer Ceratomyxa acanthuri, du nom de genre de son hôte.

\section{CERATOMYXA FISTULARIAE N. SP.}

Hôte : Fistularia petimba Lacèpède, 1803 (Fistulariidae)

Synonymes : Fistularia serrata, F. villosa

Site d'infestation : vésicule biliaire

Prévalence : $100 \%(35 / 35)$

\section{DESCRIPTION}

Les trophontes sont très polymorphes, allongés et présentent un renflement principal central. Ils sont fixés sur la paroi de la vésicule biliaire par des prolongements digitiformes. Le pansporoblaste est ovoïde, et contient deux spores (fig. 3) Les spores sont arquées avec des extrémités rétrécies et arrondies (fig. 3 et 10). Elles ont une largeur de 39,64 $\pm 0,56(38,8-40) \mu \mathrm{m}$ et un diamètre sutural de $10,25 \pm 0,60(10-12) \mu \mathrm{m}$. Les valves sont inégales. Les capsules polaires sont souvent sphériques avec un diamètre de 5,21 \pm 0,5 (4,5$5,5) \mu \mathrm{m}$ mais certaines spores ont des capsules polaires piriformes mesurant 5,15 $\pm 0,37$ (4,5-6) $\mu \mathrm{m}$ de long sur $4,21 \pm 0,41(4-4,5) \mu \mathrm{m}$. Le sporoplasme ne s'étend pas jusqu'aux extrémités des valves (fig. 3).

\section{DISCUSSION}

Cette Myxosporidie est la première décrite chez les Fistularia. Par la hauteur et la forme générale de ses spores, ainsi que le diamètre de ses capsules polaires, elle se rapproche de Ceratomyxa obtusa décrite en exURSS par Schulman (1966) chez Sphaeroides borealis. Elle se distingue néanmoins de cette espèce par la largeur de ses spores. En effet C. obtusa produit des spores de 44 à $46 \mu \mathrm{m}$ de large pour 9 à $10 \mu \mathrm{m}$ de hauteur (diamètre sutural) avec des capsules polaires sphériques de $5 \mu \mathrm{m}$ de diamètre. Nous pensons que cette espèce est nouvelle et proposons de la nommer Ceratomyxa fistulariae, du nom de genre de son hôte.

CERATOMYXA LAGOCEPHALI N. SP.

Hôte : Lagocephalus laevigatus (Linnaeus, 1766) (Tetraodontidae).

Synonyme : Lagocephalus pachycephalus 


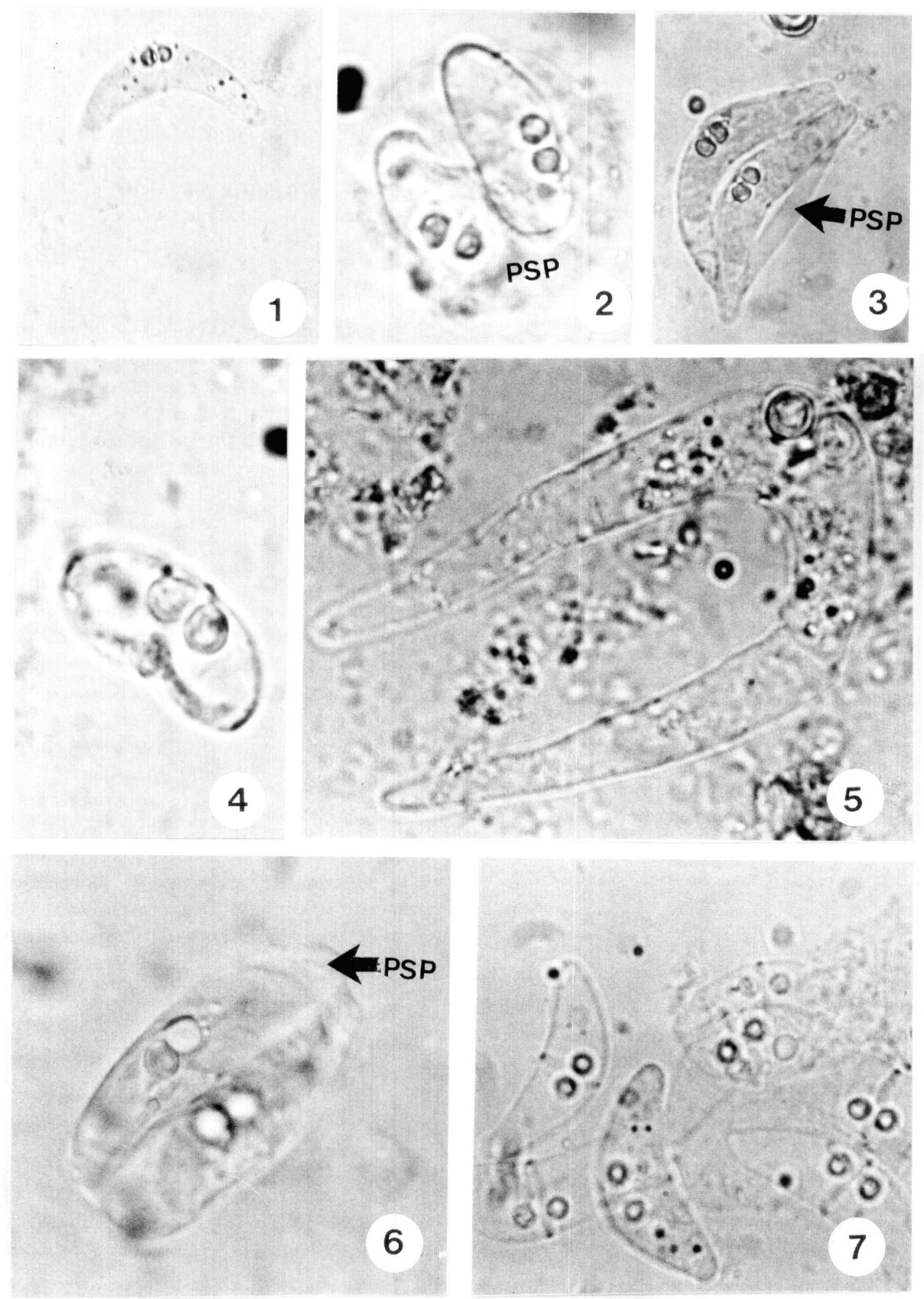

Fig. 1. - Spore vivante de Ceratomyxa trachinocephali ( $\times 1$ 200). Fig. 2. - Pansporoblaste (PSP) contenant deux spores vivantes de Ceratomyxa acanthuri $(\times 1$ 500). Fig. 3. - Pansporoblaste (PSP) contenant deux spores vivantes de Ceratomyxa fistulariae ( $\times 1200)$. Fig. 4. - Spore vivante de Ceratomyxa lagocephali $(\times 1500)$. Fig. 5. - Spore vivante de Ceratomyxa trichiuri $(\times 1$ 500). Fig. 6. - Pansporoblaste (PSP) contenant deux spores vivantes de Ceratomyxa syacii $(\times 1500)$. Fig. 7. - Spores vivantes de Ceratomyxa truncata $(\times 1500)$. 


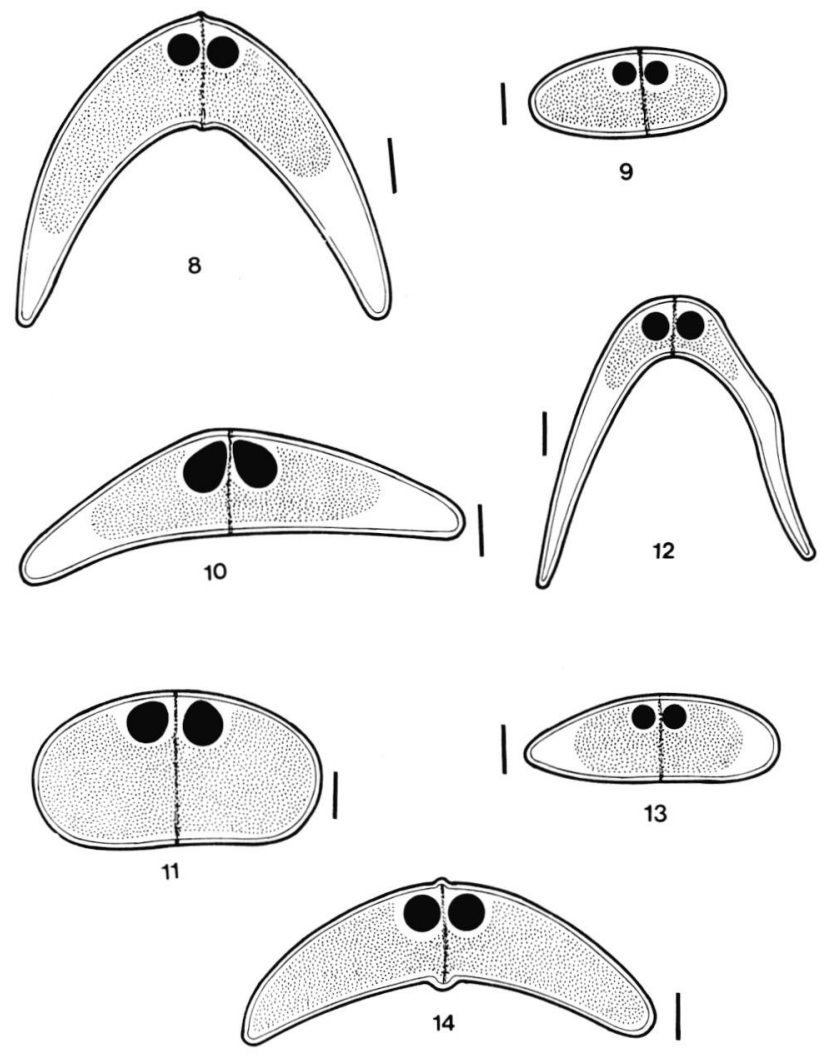

Fig. 8 à 14. - Représentations schématiques des spores des Ceratomyxa spp. étudiés. Fig. 8: C. trachinocephali $($ barre $=5 \mu \mathrm{m})$; Fig. 9: C. acanthuri (barre $=5 \mu \mathrm{m})$; Fig. 10: C. fistulariae (barre $=5 \mu \mathrm{m}) ;$ Fig. 11: C. lagocephali $($ barre $=3 \mu \mathrm{m}) ;$ Fig. 12 C. trichiuri $($ barre $=7 \mu \mathrm{m})$; Fig. 13: C. syacii $($ barre $=5 \mu \mathrm{m})$ Fig. 14: C. truncata (barre $=3 \mu \mathrm{m}$ ).

Site d'infestation : vésicule biliaire Prévalence : $25 \%(8 / 32)$

\section{DESCRIPTION}

Les spores sont légèrement arquées. Les valves sont égales et ont des extrémités arrondies (fig. 4 et 11). Ces spores mesurent 21,68 $\pm 0,6(20-22,5) \mu \mathrm{m}$ de large avec un diamètre sutural de 9,28 $\pm 0,4(9-10,50) \mu \mathrm{m}$. Les capsules polaires sont sphériques; leur diamètre est de 4,29 $\pm 0,48(3,5-4,5) \mu \mathrm{m}$. Le sporoplasme remplit toute la cavité sporale (fig. 4).

\section{Discussion}

Cette espèce présente de grandes similitudes par la forme et la largeur de ses spores avec Ceratomyxa diplodae Lubat, Radujkovic, Marquès et Bouix, 1989. En effet, $C$. diplodae produit des spores légèrement arquées mesurant $20(18-22) \mu \mathrm{m}$ de large et $6(5$ à 7) $\mu \mathrm{m}$ de diamètre sutural. Ces valves sont égales et à extrémités arrondies. Les capsules polaires mesurent $2,25 \times 2 \mu \mathrm{m}$ (Lubat et al., 1989). Cependant l'espèce décrite ici ne peut être assimilée à $C$. diplodae car le diamètre sutural de ses spores et les dimensions des capsules polaires sont nettement plus grands. Nous pensons qu'elle est nouvelle et proposons de l'appeler Ceratomyxa lagocephali du nom de genre de son hôte. Cette espèce est la première Myxosporidie décrite chez les Lagocephalus.

\section{CERATOMYXA TRICHIURI N. SP.}

Hôte : Trichiurus lepturus Linnaeus, 1758 (Trichiuridae)

Synonyme : Trichiurus japonicus

Site d'infestation : vésicule biliaire

Prévalence : $28,5 \%(10 / 35)$

\section{DESCRIPTION}

Les spores sont de grande taille. Les valves sont longues et recourbées, se rapprochant l'une de l'autre (fig. 5 et 12). Les extrémités valvaires sont rétrécies et arrondies. Les spores mesurent 99,2 \pm 0,9 (98-100) $\mu \mathrm{m}$ de large et 10,62 $\pm 0,9$ (10-12) $\mu \mathrm{m}$ de diamètre sutural. Les capsules polaires sont sphériques et mesurent 4,85 $\pm 0,2$ $(4,5-5) \mu \mathrm{m}$ de diamètre. Le sporoplasme se limite à la région médiane de la cavité sporale (fig. 5 et 12).

\section{DISCUSSION}

Les spores de l'espèce décrite ici ont des dimensions qui coincident avec celles des spores de Ceratomyxa sphaerulosa Thélohan, 1892 trouvé dans la vésicule biliaire de Mustelus canis, Galeus galeus, Clupea barengus et Scyllium canicula en France. En effet, les dimensions des spores de C. sphaerulosa sont les suivantes : largeur : 90 à $100 \mu \mathrm{m}$; diamètre sutural : 10 à $12 \mu \mathrm{m}$; capsules polaires subsphériques avec 6 à $7 \mu \mathrm{m}$ de long sur $5 \mu \mathrm{m}$ de large (Kudo, 1919). Nous ne pouvons néanmoins assimiler l'espèce de T. lepturus à C. sphaerulosa à cause de la localisation géographique de son hôte et de la forme de ses spores (les valves des spores de C. sphaerulosa ont des extrémités pointues). Nous pensons qu'elle est nouvelle et proposons de la nommer Ceratomyxa trichiuri du nom de genre de son hôte. Cette espèce est la première Myxosporidie décrite chez les Trichiurus.

\section{CERATOMYXA SYACII N. SP.}

Hôte : Syacum micrurum Ranzani, 1840 (Bohidae) Synonyme: Syacium guineensis Site d'infestation : vésicule biliaire Prévalence : $10,3 \%(6 / 58)$

\section{DESCRIPTION}

Les pansporoblastes observés sont ellipsoïdaux et disporés (fig. 6). Les spores sont droites (fig. 6 et 13) et possèdent des extrémités arrondies. Elles ont une largeur de 23,55 $\pm 0,9(22,5-25) \mu \mathrm{m}$ et un diamètre sutural de 9,33 $\pm 0,2(9-9,5) \mu \mathrm{m}$. Les valves sont 
inégales, l'une est plus courte et plus haute que l'autre. Les capsules polaires sont sphériques, très proches l'une de l'autre et ont un diamètre de 1,87 $\pm 0,1$ (1,52,0) $\mu \mathrm{m}$. Le sporoplasme occupe la totalité de la cavité sporale (fig. 6).

\section{DisCUSSION}

Cette espèce ressemble à Ceratomyxa monospora Davis, 1917, Ceratomyxa undulata Davis, 1917 et à Ceratomyxa inaequalis Doflein, 1898 par la forme et la largeur de ses spores (Kudo, 1919; Lubat et al., 1989) (tableau I). Elle diffère néanmoins de ces trois espèces par le diamètre sutural de ses spores et par le diamètre des capsules polaires. Nous pensons que cette espèce est nouvelle et proposons de la nommer Ceratomyxa syacii du nom de genre de son hôte.

\section{CeratomyXa truncata Thélohan, 1895}

Hôte : Sardinella maderensis Lowe, 1839 et Sardinella aurita Valenciennes, 1847 (Clupeidae)

Site d'infestation : vésicule biliaire

Prévalences : 4,1\%(3/72) chez $S$. maderensis et 1,6 $\%(1 / 61)$ chez S. aurita.

\section{DESCRIPTION}

Les spores sont arquées avec des valves égales aux extrémités rétrécies et arrondies (fig. 7 et 14). Elles ont une largeur de $26,05 \pm 0,7(25,5-27) \mu \mathrm{m}$ et un diamètre sutural de $6,08 \pm 0,16(5-7) \mu \mathrm{m}$. Les capsules polaires sont sphériques, de petite taille et mesurent 2,17 $\pm 0,1$ $(2-2,25) \mu \mathrm{m}$ de diamètre. Le sporoplasme s'étend dans toute la cavité sporale (fig. 7).

\section{Discussion}

Cette espèce produit des spores dont les dimensions et la forme correspondent exactement à celles des spores de Ceratomyxa truncata Thélohan, 1895 parasite de la vésicule biliaire de Chpea pilchardus en France et en Italie. En effet, les spores de C. truncata ont des valves courtes, tronquées et mesurent $25 \mu \mathrm{m}$ de large et $5 \mu \mathrm{m}$ de haut (diamètre sutural) (Kudo, 1919). Par ailleurs son hôte appartient à la même famille que les hôtes de notre Ceratomyxa. Nous pensons être en présence de la même espèce. $S$. maderensis et $S$. aurita sont des hôtes nouveaux.

\section{CONCLUSIONS}

u total sept espèces de Myxosporidies, dont
six sont nouvelles pour la science, ont été
trouvées chez des poissons des côtes sénégalaises. Ces Myxosporidies sont colozö̈ques car elles n'infestent que la vésicule biliaire de leurs hôtes. Il semble que cet organe soit le site de prédilection des Ceratomyxa spp. Lom et Dykovà (1992) avaient d'ailleurs rapporté que les Ceratomyxa sont rarement histozoïques.

Selon de nombreux auteurs, les Myxosporidies coelozoïques ont une action pathogène négligeable. Cependant Sakiti (1982) et Fomena (1986) pensent que ces Myxosporidies peuvent contribuer au blocage de l'écoulement de la bile, ce qui peut perturber la digestion chez les poissons hôtes et entraîner le ralentissement de leur croissance ou leur mort. Selon Sakiti (1982), Ceratomyxa dicentrarchi forme des calculs qui obstruent le canal d'écoulement de la bile chez Dicentrarchus labrax. Fomena (1986) signale que Myxidium camerounensis forme des trophontes de grande taille qui bloquent l'écoulement de la bile chez Neolebias ansorgei. Nous n'avons observé aucun cas d'obstruction du canal d'écoulement de la bile chez les poissons parasités que nous avons examinés.

\begin{tabular}{|c|c|c|}
\hline Espèces & Caractères des spores & $\begin{array}{l}\text { Hôtes et sites } \\
\text { d'infestation }\end{array}$ \\
\hline $\begin{array}{l}\text { Ceratomyxa monospora } \\
\text { Davis, } 1917\end{array}$ & $\begin{array}{l}\text { En forme de croissant } 18-25 \mu \mathrm{m} \times 5-6 \mu \mathrm{m} \text {. } \\
\text { Valves cylindriques et extrémités arrondies. }\end{array}$ & Perprilus alepidotus (vésiculaire biliaire) \\
\hline $\begin{array}{l}\text { Ceratomyxa undulata } \\
\text { Davis, } 1917\end{array}$ & $\begin{array}{l}\text { En forme de croissant } 22-25 \mu \mathrm{m} \times 6 \mu \mathrm{m} \text {. } \\
\text { Valves cylindriques et extrémités arrondies. }\end{array}$ & Ancylopsetta quadrocellata (vésicule biliaire) \\
\hline $\begin{array}{l}\text { Ceratomyxa inaequalis } \\
\text { Doflein, } 1898\end{array}$ & $\begin{array}{l}\text { Ovales, extrémités arrondies : } \\
25(23,5-31) \mu \mathrm{m} \times 5,5(5-6) \mu \mathrm{m} \text {. } \\
\text { Valves souvent inégales. Capsules polaires : } 2,5 \mu \mathrm{m} \text {. }\end{array}$ & $\begin{array}{l}\text { Symphodus (Crenilabrus) mediterraneus } \\
\text { S. tincta, S. pavo (vésicule biliaire) }\end{array}$ \\
\hline $\begin{array}{l}\text { Ceratomyxa syacii } \\
\text { (présent travail) }\end{array}$ & $\begin{array}{l}\text { Ellipsoïdes, extrémités arrondies : } \\
22,55 \pm 0,9(22,5-25) \mu \mathrm{m} \times 9,33 \pm 0,2(9-9,5) \mu \mathrm{m} . \\
\text { Valves inégales. Capsules polaires : } \\
1,87 \pm 0,1(1,8-2,02) \mu \mathrm{m} .\end{array}$ & Syacium micrurum (vésicule biliaire) \\
\hline
\end{tabular}

Tableau I. - Comparaison entre les Ceratomyxa se rapprochant de Ceratomyxa syacii par la forme et la largeur de leurs spores. 


\section{REFERENCES}

Abolarin M.O. A new species of Henneguya (Myxosporidia, Protozoa) from West african catfish, Clarias lazera. Val., with a review of the genus Henneguya Thélohan, 1892. Afr. J. Trop. Hydrobiol. Fish, 1971, 1, 93-105.

Abolarin M.O. Myxobolus tilapiae sp. n. (Protozoa: Myxosporidia) from three species of freshwater Tilapia in Nigeria. Journal of West African Science Association, 1974, 19, 109-114.

Baker J.R. Tree new species of Myxozoma (Prozoa: Myxosporidia) from East african freshwater fish. Parasitology, $1963,53,285-292$.

Bartholomew J.L., Smith C.E., Rohovec J.C. \& Fryer J.L. Characterisation of a host response to the myxosporean parasite, Ceratomyxa shasta (Noble), by histology, scanning electron microscopy and immunological techniques. Journal of Fish diseases, 1989, 12, 509-522.

Berrebi P. Omniprésence d'une Myxosporidie chez les barbeaux marocains (Poissons, Téléostéens, Cyprinidés). Bulletin de l'Institut Scientifique, Rabat, 1980, 4, 191-192.

Dubina V.R. \& Isakov L.S. New species of Myxosporidians from the gallbladder of bathial fishes. Parasitologiya, 1976, $10,556-560$.

Faisal M. \& Shalaby S.I. Myxosoma tilapiae as a new species (Myxosoma: Myxosporea) in wild Oreochromis niloticus in lower Egypt. Egyptian Journal of Veterinary Science, 1987, 1, 73-86.

Fantham H.B. Some parasitic Protozoa found in South Africa. III. South African Journal of Sciences, 1930, 27, 376-390.

Fomena A. Contribution à l'étude des Myxosporidies (Protozoa, Myxozoa) parasites des poissons d'eau douce du Sud-Cameroun : systématique, ultrastructure, relations hôte-parasite. Thèse de Doctorat de $3^{e}$ cycle, Université de Yaoundé, 1986, 276 p.

Fomena A. \& Boulx G. Contribution à l'étude des Myxosporidies des poissons d'eau douce du Sud-Cameroun. I. Espèces nouvelles du genre Myxidium Bütschli, 1882. Acta Tropica, 1986, 43, 319-333.

Fomena A. \& Boutx G. Contribution à l'étude des Myxosporidies des poissons d'eau douce du Sud-Cameroun. III. Espèces nouvelles des genres Henneguya Thélohan, 1892 et Thelohanellus Kudo, 1933. Revue de Zoologie Africaine, $1987,101,43-53$.

Fomena A., Boutx G. \& Birgi E. Contribution à l'étude des Myxosporidies des poissons d'eau douce du Sud-Cameroun. II. Espèces nouvelles du genre Myxobolus Bütschli, 1882. Bulletin de l'IFAN, 1985, (sér. A), 46, 167-192.

Fomena A., Marques A. \& Bouix G. Myxosporidea (Myxozoa) of Oreochromis niloticus (Linnaeus, 1757) (Teleost, Cichlidae) in fish-farming pools at Melen (Yaoundé, Cameroon, Central Africa). Journal of African Zoology, 1993, $107,45-56$.

Kabre G.G., Sakiti N.G., Marques A. \& Sawadogo L. Myxobolus comei n. sp. et Myxobolus burkinei n. sp., nouvelles Myxosporidies histozoïques chez deux Téléostéens, Clarias anguillaris et Labeo coubie, capturés dans les pêcheries du Burkina Faso (Afrique de l'Ouest). Bulletin de l'IFAN Cheikh Anta Diop, Dakar, 1995, sér. A, 48, 49-55.
Kostoingue B. \& Toguebaye B.S. Le genre Myxobolus (Myxozoa, Myxosporea) chez les poissons d'eau douce du Tchad avec la description de trois nouvelles espèces. Bulletin de l'IFAN Cheikh Anta Diop, Dakar, 1994, sér. A, 47 , 63-71.

Kudo R. Studies on Myxosporidia. A synopsis of genera and species of Myxosporidia. Illinois Biological Monographs, $1919,5,1-265$.

Lom J. Phylum Myxozoa. In: Handbook of Protoctista. L. Margulis, J.O. Corlis, M. Melkonian, D.J. Chapman (Eds), Jones and Barlett Publishers, Boston, 1990, p. 36-52.

Lom J. \& Dykovà I. Protozoan parasites of fishes. Development in aquaculture and fisheries science, 1992, 26, 1-315.

Lubat V., Radujkovic B., Marques A. \& Boutx G. Parasites des poissons marins du Monténégro : Myxosporidies. Acta Adriatica, 1989, 30, 31-50.

Molnàr K. Ceratomyxa hungarica n. sp. and Chloromyxum proterorbini n. sp. (Myxozoa: Myxosporea) from the fresh water goby Proterorhinus marmoratus (Pallas). Systematic Parasitology, 1992, 22, 25-31.

Obiekezie A.I. \& ENyenihi U.K. Henneguya chrysichthyi sp. n. (Protozoa: Myxozoa) from the gills of estuarines catfish Chrysichthys nigrodigitatus (Lacépède) (Pisces: Bagridae) in Nigeria. Revue de Zoologie Africaine, 1988, 102, 33-42.

OBIEKEZIE A.I. \& OKAEME A.N. Myxobilatus accessobranchialis n. sp. (Protozoa: Myxozoa) from the accessory breathing organ of cultured Heterobranchus bidorsalis Geoffroy Saint Hilaire, 1809. Arch. Protistenkd., 1987, 134, 409-411.

Obiekezie A.I. \& Okaeme A.N. Myxosporean (Protozoa) infections of cultured Tilapias in Nigeria. Journal of African Zoology, 1990, 104, 77-79.

Obiekezie A.I. \& SсHмAн G. Henneguya laterocapsula Landsberg, 1987 (Myxosporea, Myxozoa) in cultured hybrid african catfish: ultrastructure of the parasite-host interface. European Journal of Protistology, 1993, 29, 38-41.

PAPERNA I. Occurrence of Cnidospora infection in freshwater fishes in Africa. Bulletin de l'IFAN, 1973, 33 (sér. .A), 509-527.

Peters H.M. Sporozoon cysts, the basis of purported " MouthBrooding " in the african labyrinthine fish, Ctenopoma multispinis (Pisces: Anabantidae). Copeia, 1971, 1, 185.

SAKITI N.G. Recherches ultrastructurales et biologiques sur quelques Myxosporidies parasites de Serranidés (Poissons, Téléostéens). Thèse de Doctorat de $3^{e}$ cycle, Université des Sciences et Techniques du Languedoc, Montpellier, 1982, $147 \mathrm{p}$.

Sakiti N., Blanc E., Marques A. \& Boutx G. Myxosporidies (Myzozoa, Myxosporea) du genre Myxobolus Bütschli, 1882, parasite de poissons Cichlidae du Lac Nokoué au Bénin (Afrique de l'Ouest). Journal of African Zoology, 1991, 105, 173-186.

Schulman S.S. Myxosporidies de la Faune d'URSS. Nauka, Moscou, 1966, 504 p. (en russe)

SIAU Y. Myxosporidies de Synodontis ansorgii Bouleng. Ann. et Mag., 1911 et Eleotris (Kribia) kribiensis Boulenger, 1964, Poissons des eaux saumâtres de la lagune de PortoNovo (Dahomey). Bulletin de la Société de Zoologie de France, 1971, 96, 563-570.

Reçu le 8 janvier 1996 Accepté le 11 mai 1996 